Draft VERSion MAY 12, 2019

Preprint typeset using $\mathrm{L}^{\mathrm{A}} \mathrm{T}_{\mathrm{E}} \mathrm{X}$ style emulateapj v. 01/23/15

\title{
CONSTRAINTS ON THE PROGENITOR SYSTEM OF SN 2016GKG FROM A COMPREHENSIVE STATISTICAL ANALYSIS
}

\author{
Niharika Sravan, Pablo Marchant, Vassiliki Kalogera, and Raffaella Margutti \\ Center for Interdisciplinary Exploration and Research in Astrophysics (CIERA) \\ and \\ Department of Physics and Astronomy \\ Northwestern University, 2145 Sheridan Road, Evanston, IL 60208, USA \\ Draft version May 12, 2019
}

\begin{abstract}
Type IIb supernovae ( $\mathrm{SNe}$ ) present a unique opportunity for understanding the progenitors of stripped-envelope (SE) SNe as the stellar progenitor of several Type IIb SNe have been identified in pre-explosion images. In this paper, we use Bayesian inference and a large grid of non-rotating solar-metallicity single and binary stellar models to derive the associated probability distributions of single and binary progenitors of the Type IIb SN $2016 \mathrm{gkg}$ using existing observational constraints. We find that potential binary star progenitors have smaller pre-SN hydrogen-envelope and helium-core masses than potential single-star progenitors typically by $0.1 M_{\odot}$ and $2 M_{\odot}$, respectively. We find that, a binary companion, if present, is a main-sequence or red-giant star. Apart from this, we do not find strong constraints on the nature of the companion star. We demonstrate that the range of progenitor helium-core mass inferred from observations could help improve constraints on the progenitor. We find that the probability that the progenitor of SN $2016 \mathrm{gkg}$ was a binary is $22 \%$ when we use constraints only on the progenitor luminosity and effective temperature. Imposing the range of pre-SN progenitor hydrogen-envelope mass and radius inferred from SN light-curves the probability the progenitor is a binary increases to $44 \%$. However, there is no clear preference for a binary progenitor. This is in contrast to binaries being the currently favored formation channel for Type IIb SNe. Our analysis demonstrates the importance of statistical inference methods to constrain progenitor channels.

Subject headings: binaries: general - stars: massive - supernovae: general - supernovae: individual
\end{abstract}

(SN 2016gkg)

\section{INTRODUCTION}

The mechanisms driving the stripping of the progenitor stars of stripped-envelope (SE) supernovae (SNe) remain an open research question. Currently, close binary interactions, stellar winds, and nuclear burning instabilities are leading candidates to explain the mass loss (e.g. Claeys et al. 2011; Arnett \& Meakin 2011; Groh et al. 2013 Smith 2014: Soker 2017). Among SE SNe, Type IIb $\mathrm{SNe}$ explode with a low-mass residual hydrogen-envelope (e.g., Podsiadlowski et al. 1992; Woosley et al. 1994) and initially exhibit prominent hydrogen spectral features that weaken and disappear in the weeks following the explosion. The progenitors of five Type IIb SNe have been identified in pre-explosion images: 1993J (Aldering et al. 1994), 2008ax (Folatelli et al. 2015), 2011dh (Maund et al. 2011, Van Dyk et al. 2011), 2013df (Van Dyk et al. 2014), and 2016gkg (Kilpatrick et al. 2017). Furthermore, there is evidence for the presence of binary companions to the progenitors of SNe 1993J and 2011dh (Fox et al. 2014; Folatelli et al. 2014). This makes Type IIb SNe ideal candidates to test theories of binary evolution.

SN 2016gkg was discovered on 2016 September 20.18 UT in NGC 613. Kilpatrick et al. (2017) identified a source in pre-explosion Hubble Space Telescope (HST) images as its progenitor and inferred its luminosity and effective temperature. Tartaglia et al. (2017) found an additional source and concluded they could not favor either source as the progenitor star. They also found different magnitudes for the common source. The properties for this source inferred by Tartaglia et al. (2017) are consistent (within 1 and $3 \sigma$ in luminosity and effective temperature, respectively) with those inferred by Kilpatrick et al. (2017). Therefore, for simplicity, we adopt the constraints of Kilpatrick et al. (2017). Arcavi et al. (2017) fit analytic models to the light curve of SN 2016gkg and derived a radius and residual hydrogen-envelope mass for the progenitor star (see Table 1).

In this paper, given observational constraints on its progenitor properties, we use Bayesian inference to derive the distribution of properties of potential progenitor systems (both singles and binaries) of SN 2016gkg. We also calculate the probability that the progenitor was a binary. We assume that the constraints derived by Kilpatrick et al. (2017) corresponds to the progenitor. We discuss the effect of using the pre-SN progenitor hydrogen-envelope and helium-core mass constraints to distinguish between single and binary progenitor channels.

This paper is organized as follows. In Section 2 we briefly describe our models and method. In Section 3 we discuss results for the distribution of potential progenitor systems (both singles and binaries) of SN 2016gkg. We summarize our results and conclude in Section 4 .

\section{METHOD}

\subsection{Single and Binary Star Models}


TABLE 1

Properties of Detected SN 2016gkg Progenitor

\begin{tabular}{cccc}
\hline \hline $\log _{10}\left(L / L_{\odot}\right)$ & $T_{\text {eff }} / \mathrm{K}$ & $R / R_{\odot}$ & $M_{\text {env }} / M_{\odot}$ \\
\hline $5.14_{-0.14}^{+0.36}{ }^{a}$ & $9500_{-1033}^{+3700}{ }^{a}$ & $40-150^{b}$ & $0.02-0.4^{b}$ \\
\hline
\end{tabular}

REFERENCES. - ${ }^{a}$ Kilpatrick, C. D., private communication (uncertainties are one-third of $3 \sigma$, see Section 2.2); $b$ Arcavi et al. 2017).

We compute a large grid of non-rotating solarmetallicity 11 single and binary star models with Modules for Experiments in Stellar Astrophysics (MESA ${ }^{2}$, Paxton et al. 2011, 2013, 2015). We briefly summarize the models in what follows.

We start the evolution of the star(s) at the zero-age main sequence (ZAMS). We stop the evolution if any of the following conditions are met: the carbon mass fraction at (any) star's center is lower than $10^{-6}$, the hydrogen-envelope mass of any star drops below $0.01 M_{\odot}$ (in which case we assume the system is a completely stripped Type Ibc progenitor), or, in binaries, the accretor overfills its Roche-lobe. We assume the surface properties of the star at carbon depletion match those of the pre-supernova progenitor star. This is because the thermal timescale of the envelope is large compared to the time between carbon depletion and iron core-collapse. We note however that it has recently been proposed that waves could efficiently transport energy outwards during core neon and oxygen burning, potentially producing outbursts and large changes in the progenitor surface luminosity and effective temperature months or years prior to the explosion Quataert \& Shiode 2012, Shiode \& Quataert 2014 Fuller 2017).

We use the basic.net, approx21.net, and co_burn .net nuclear networks in MESA. We adopt the standard mixing-length theory and the Ledoux criterion to model convection, with $\alpha_{\mathrm{MLT}}$ set to 1.5. When convective regions approach the Eddington limit, the efficiency of convection is enhanced ${ }^{3}$ (Paxton et al. 2013). To account for the nonzero momentum of a convective element at the Hydrogen burning convective core boundary, we extend this region by 0.335 of the pressure scale height (Brott et al. 2011). We adopt the value of dimensionless free parameter for semi-convection, $\alpha_{\mathrm{sc}}$, to be 1.0 (Yoon et al. 2006). We use radiative opacity tables from the OPAL project (Iglesias \& Rogers 1996). We adopt surface effective temperature and abundance dependent stellar wind prescriptions. When $T_{\text {eff }}>10^{4} \mathrm{~K}$, we adopt the prescription of Vink et al. (2001) if the surface hydrogen mass fraction $>0.4$ and Nugis \& Lamers (2000) otherwise. If $T_{\text {eff }}<10^{4} \mathrm{~K}$ we adopt the prescription of de Jager et al. (1988).

We use the model of Kolb \& Ritter (1990) to calculate the mass transfer rate due to Roche-lobe overflow (RLO)

\footnotetext{
1 We choose the value of solar metallicity $\left(Z_{\odot}\right)$ to be 0.02 . The metallicity of the host galaxy of SN 2016gkg is $0.012 \pm 0.004$ (Kilpatrick et al. 2017). See Conclusions for a discussion on the effect of metallicity.

2 Release 9575.

3 The treatment of these regions is a subject of debate and stellar evolution calculations during these phases are uncertain.
}

in our binary star models. The efficiency of mass transfer (the ratio of mass accreted by the secondary to the mass transferred via RLO by the primary), $\epsilon$, is assumed to be constant during the evolution. The mass not accreted is assumed to be lost as stellar winds. Stellar winds carry away with them the specific angular momentum of the corresponding component. All orbits are assumed to be circular.

We compute single-star models with initial mass, $\log _{10}\left(M_{\mathrm{ZAMS}} / M_{\odot}\right)=1.28-1.40\left(M_{\mathrm{ZAMS}} / M_{\odot} \sim 19-\right.$ $25)$ in intervals of 0.0005 dex and binary star models with initial primary mass, $\log _{10}\left(M_{\mathrm{ZAMS}, 1} / M_{\odot}\right)=1.0-$ $1.4\left(M_{\mathrm{ZAMS}, 1} / M_{\odot} \sim 10-25\right)$ in intervals of $0.02 \mathrm{dex}$, initial mass ratio, $q_{\mathrm{ZAMS}} \equiv M_{\mathrm{ZAMS}, 2} / M_{\mathrm{ZAMS}, 1}=0.225$ - 0.975 in intervals of 0.05 , and initial orbital period, $\log _{10}\left(P_{\text {orb }} / \mathrm{d}\right)=2.5-3.8\left(P_{\text {orb }} / \mathrm{d} \sim 316-6310\right)$ in intervals of 0.02 dex 4 We choose this parameter space based on a broader scan. We compute the models for $\epsilon$ $=0.5$ and 0.1. Models that reach core carbon exhaustion $\left(C_{\text {center }} \leq 10^{-6}\right)$ with less than $1 M_{\odot}$ (but greater than $\left.0.01 M_{\odot}\right)$ of residual hydrogen-envelope are defined as Type IIb SN progenitors. This criterion is a conservative choice as residual hydrogen envelope masses of Type IIb SNe with detected progenitors are less than $0.5 M_{\odot}$ (Woosley et al. 1994). Type IIb SNe with detected progenitors represent those with the most massive envelopes: progenitors with smaller envelopes are compact (Yoon et al. 2017) and thus harder to detect. Moreover, the cooling envelope feature in Type IIb SN light curves decrease with decreasing radius (Moriya et al. 2016) and envelope mass making compact Type IIb SNe harder to detect.

\subsection{Statistical Method}

We use Bayesian inference to derive the distribution of the potential progenitors (and their binary companions) of SN 2016gkg. We adopt $3 \sigma$ (see below for reasoning) luminosity and effective temperature constraints on the progenitor as derived from observations of the progenitor system before explosion (Kilpatrick, C. D., private communication). We assume that luminosity and effective temperature are independent variables for simplicity, though this assumption is not accurate. We do not apply the progenitor hydrogen-envelope mass and radius constraints derived from the SN light-curves as these are model-dependent. However, we discuss the implications of applying them later.

For each Type IIb SN progenitor model (see above for definition) we compute a posterior probability $(P)$ :

$$
P\left(\vec{\theta}_{\text {mod }} \mid \vec{X}_{\text {obs }}\right)=\mathcal{L}\left(\vec{X}_{\text {obs }} \mid \vec{\theta}_{\text {mod }}\right) P\left(\vec{\theta}_{\text {mod }}\right),
$$

4 MESA inlists used for these can be found at https://github. com/orlox/mesa_input_data/tree/master/2017_IIb 

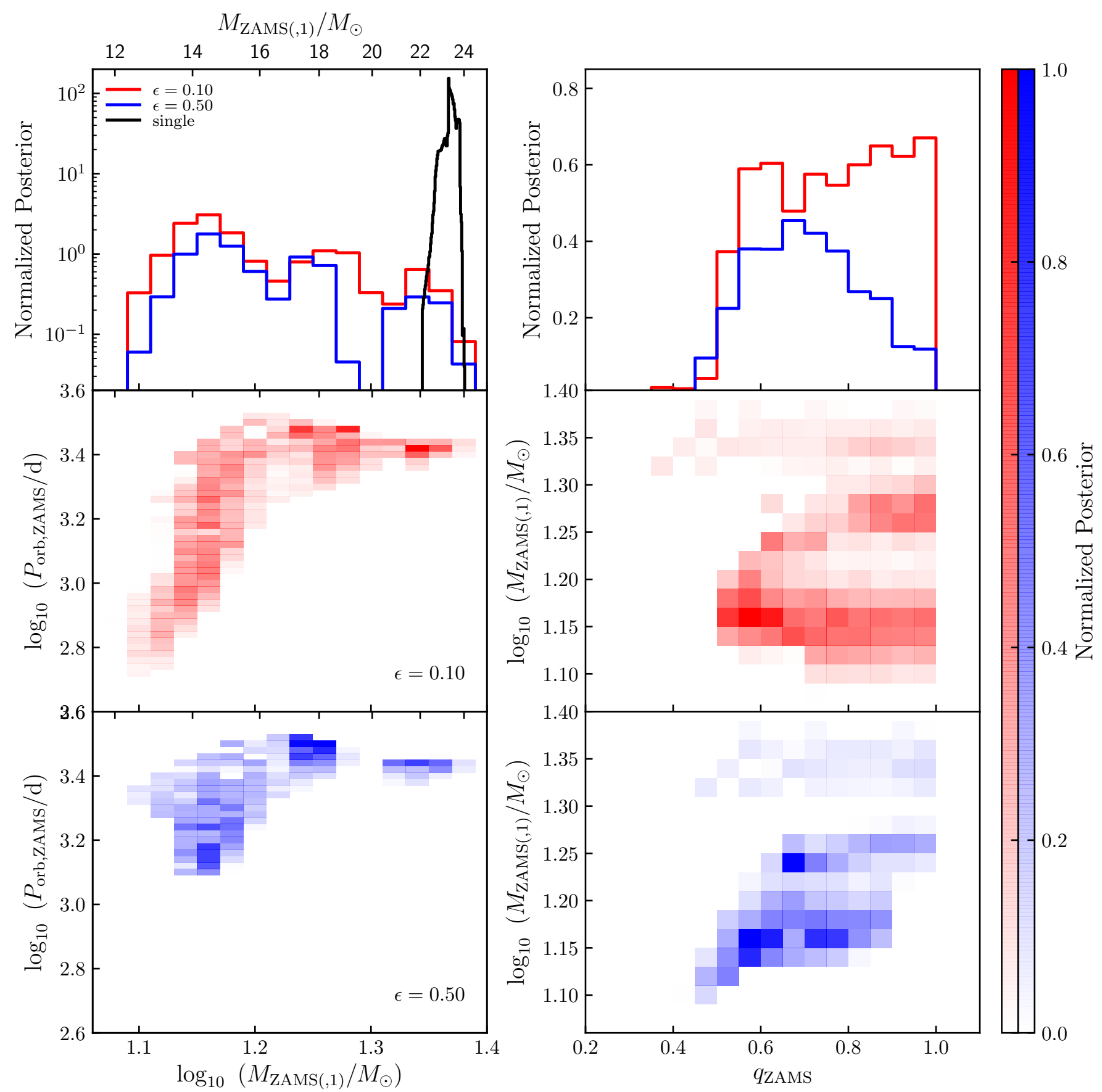

FIG. 1. - Posterior probability distributions of the parameter space of potential single and binary star progenitors of SN $2016 \mathrm{gkg}$. The top panel shows the distribution of initial (primary) mass $\left(M_{\mathrm{ZAMS}(, 1)}\right.$, left) and, for binaries, initial mass ratio $\left(q_{\mathrm{ZAMS}} \equiv M_{\mathrm{ZAMS}, 2} / M_{\mathrm{ZAMS}, 1}\right.$, right) of potential single (black) and binary star progenitors with $\epsilon=0.1$ (red) and 0.5 (blue). The histograms show the total posterior probability in each bin. The histograms in the top left panel have been rescaled such that areas under them reflect the probability of a single or binary star progenitor of SN $2016 \mathrm{gkg}$. The middle and bottoms panels show 2-D distributions of $P_{\text {orb }}$ and $M_{\mathrm{ZAMS}(, 1)}$ (left) and $M_{\mathrm{ZAMS}(, 1)}$ and $q_{\mathrm{ZAMS}}$ (right) of potential binary star progenitors with $\epsilon=0.1$ (middle) and 0.5 (bottom).

modulo the standard normalization constant in Bayes' theorem. The model parameters, $\vec{\theta}_{\text {mod }}$, are the initial single (initial mass) or binary (initial primary mass, mass ratio, and orbital period) star progenitor properties and eventually determine the pre-SN progenitor properties, $\vec{X}_{\text {mod }}$, i.e., $\vec{X}_{\text {mod }}\left(\vec{\theta}_{\text {mod }}\right)$. For each individual observable quantity $X_{\mathrm{obs}, \mathrm{i}}$ (the $i$-th component of vector $\vec{X}_{\mathrm{obs}}$ ) with mean, $\mu_{\mathrm{i}}$, and uncertainties, $\sigma_{+}-, \mathrm{i}$, we adopt the split normal distribution for the likelihood

$$
\begin{aligned}
\mathcal{L}\left(\mu_{\mathrm{i}}, \sigma_{+, \mathrm{i}}, \sigma_{-, \mathrm{i}} \mid X_{\text {mod }, \mathrm{i}}\right)= & \sqrt{\frac{2}{\pi}} \frac{1}{\sigma_{-, \mathrm{i}}+\sigma_{+, \mathrm{i}}} \times \\
& \exp \left(-\frac{\left(X_{\text {mod }, \mathrm{i}}-\mu_{\mathrm{i}}\right)^{2}}{2 \sigma_{\mathrm{i}}^{2}}\right)
\end{aligned}
$$



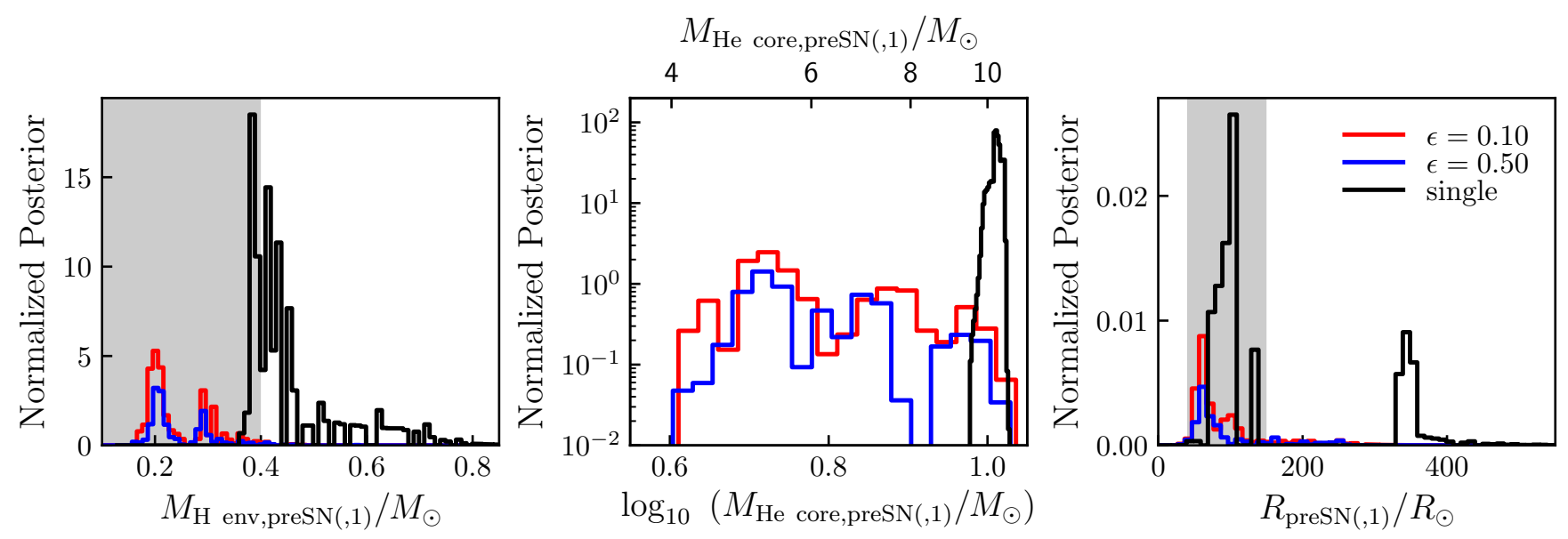

FIG. 2.- Distributions of pre-SN properties of potential single (black) and binary $(\epsilon=0.1$ (red) and 0.5 (blue)) star progenitors of SN 2016gkg: progenitor hydrogen-envelope mass $\left(M_{\mathrm{H} \text { env,preSN(,1) }}\right.$, left), progenitor helium-core mass $\left(M_{\mathrm{He}}\right.$ core,preSN(,1), middle), and progenitor radius $\left(R_{\text {preSN(,1) }}\right.$, right). The histograms show the total posterior probability in each bin and have been rescaled such that areas under them reflect the probability of a single or binary star progenitor of SN 2016gkg. Grey shaded regions indicate constraints derived from light-curves (see Table 1). Pre-SN hydrogen-envelope and helium-core mass for potential binary star progenitors are smaller than for potential single-star progenitors typically by $0.1 M_{\odot}$ and $2 M_{\odot}$, respectively. Constraints on the progenitor hydrogen-envelope mass and radius from light-curves increase the likelihood of a binary progenitor of SN $2016 \mathrm{gkg}$ from $22 \%(13 \%)$ to $44 \%(28 \%)$ for $\epsilon=0.1(0.5)$.

where $\sigma_{\mathrm{i}}=\sigma_{-, \mathrm{i}}$ when $X_{\bmod , \mathrm{i}}<\mu_{\mathrm{i}}$ and $\sigma=\sigma_{+, \mathrm{i}}$ when $X_{\text {mod,i }} \geq \mu_{\mathrm{i}}$. For each individual observable quantity $X_{\text {obs,i }}$ with a range of values, we adopt a flat distribution for the likelihood. Thus,

$$
\mathcal{L}\left(\vec{X}_{\text {obs }} \mid \vec{\theta}_{\text {mod }}\right)=\prod_{\mathrm{i}} \mathcal{L}\left(\mu_{\mathrm{i}}, \sigma_{+, \mathrm{i}}, \sigma_{-, \mathrm{i}} \mid X_{\text {mod }, \mathrm{i}}\right)
$$

Observational uncertainties are not necessarily distributed as a normal distribution and this form of the likelihood function is just an approximation. In particular, the $1 \sigma$ range for luminosity and effective temperature from Kilpatrick et al. (2017) is much wider than one-third of their $3 \sigma$ range (Kilpatrick, C. D., private communication). To avoid artificially extending the range of uncertainty in the observations, we adopt $\sigma_{+}$and $\sigma_{-}$to be one-third of the respective $3 \sigma_{+}$and $3 \sigma_{-}$values instead of the $1 \sigma_{+/-}$values in Kilpatrick et al. (2017).

The prior probability $P\left(\vec{\theta}_{\text {mod }}\right)$ is computed for the range $\left[\vec{\theta}_{\text {mod }}-\Delta \vec{\theta}_{\text {mod }} / 2, \vec{\theta}_{\text {mod }}+\Delta \vec{\theta}_{\text {mod }} / 2\right]$. For a singlestar with initial mass, $\log _{10} M_{\mathrm{ZAMS}}$,

$$
P\left(\vec{\theta}_{\mathrm{mod}}\right)=\left(1-f_{\mathrm{bin}}\right) P\left(\log _{10} M_{\mathrm{ZAMS}}\right)
$$

and for a stellar binary with initial primary mass, $\log _{10} M_{\mathrm{ZAMS}, 1}$, initial mass ratio, $q_{\mathrm{ZAMS}}$, and initial orbital period, $P_{\text {orb }}$,

$$
\begin{array}{r}
P\left(\vec{\theta}_{\mathrm{mod}}\right)=f_{\mathrm{bin}} P\left(\log _{10} M_{\mathrm{ZAMS}, 1}\right) \\
P\left(q_{\mathrm{ZAMS}}\right) \\
P\left(\log _{10} P_{\mathrm{orb}}\right)
\end{array}
$$

where, $f_{\text {bin }}$ is the fraction of stars in binaries.

We assume $f_{\text {bin }}$ to be a constant and independent of $M, q$, and $P_{\text {orb }}$. The distribution of $M$ is taken to be the Salpeter Initial Mass Function (IMF, Salpeter 1955)

$$
f(M)=M^{-\alpha}
$$

We assume that the minimum ZAMS mass needed to undergo core-collapse is $8 M_{\odot}$ (Woosley et al. 2002). We adopt a power-law distribution for the initial mass ratio, $q$,

$$
f(q)=q^{\beta}
$$

This distribution is assumed to be followed for $q>0.2$ (Kobulnicky et al. 2014). Finally, the distribution of initial orbital period, $P_{\text {orb }}$, is chosen according to Kobulnicky et al. (2014)

$$
f\left(\log _{10} P_{\text {orb }}\right)=\left(\log _{10} P_{\text {orb }}\right)^{\gamma}
$$

This distribution is assumed to hold 5 for $0.15<$ $\log _{10}\left(P_{\text {orb }} / \mathrm{d}\right)<4$.

\section{RESULTS}

We compute posterior probabilities for SN 2016gkg using our model Type IIb SN progenitors (see Section 2.1 for definition) using the method described above. Unless otherwise mentioned, we assume $f_{\text {bin }}=0.5, \alpha=2.3$ (Salpeter 1955), $\beta=-1$, and $\gamma=-0.22$ (Kobulnicky et al. 2014).

Some binary star models experience very little interaction, transferring only small amounts of mass when the primary star's atmosphere Roche-lobe overflows. Therefore their evolution largely resembles that of single stars. We therefore require that primaries transfer at least $1 \%$ of their initial mass in RLO to qualify as 'binary' progenitors. The exact choice for this number does not affect our results significantly; lowering it by an order of magnitude adds some $\gtrsim 22 M_{\odot}$ mass binaries with net posterior probabilities $\lesssim 3 \%$ more for our fiducial priors.

In figure 1 we show the distribution of the parameter space of potential single and binary star progenitors of SN 2016gkg. There are three peaks in the distribution of initial primary mass for binary star progenitors. The low mass peak is favored by the prior on initial primary mass (Eq. 6), the middle peak is due to the likelihood for $T_{\text {eff }}$, and the high mass peak results from mildly interacting binaries with relatively undisturbed primaries

5 The upper limit for the validity of this distribution is 2000 days (Kobulnicky et al.2014). However, due to poor constraints for wide binaries we assume that this distribution holds up to 10,000 days. 


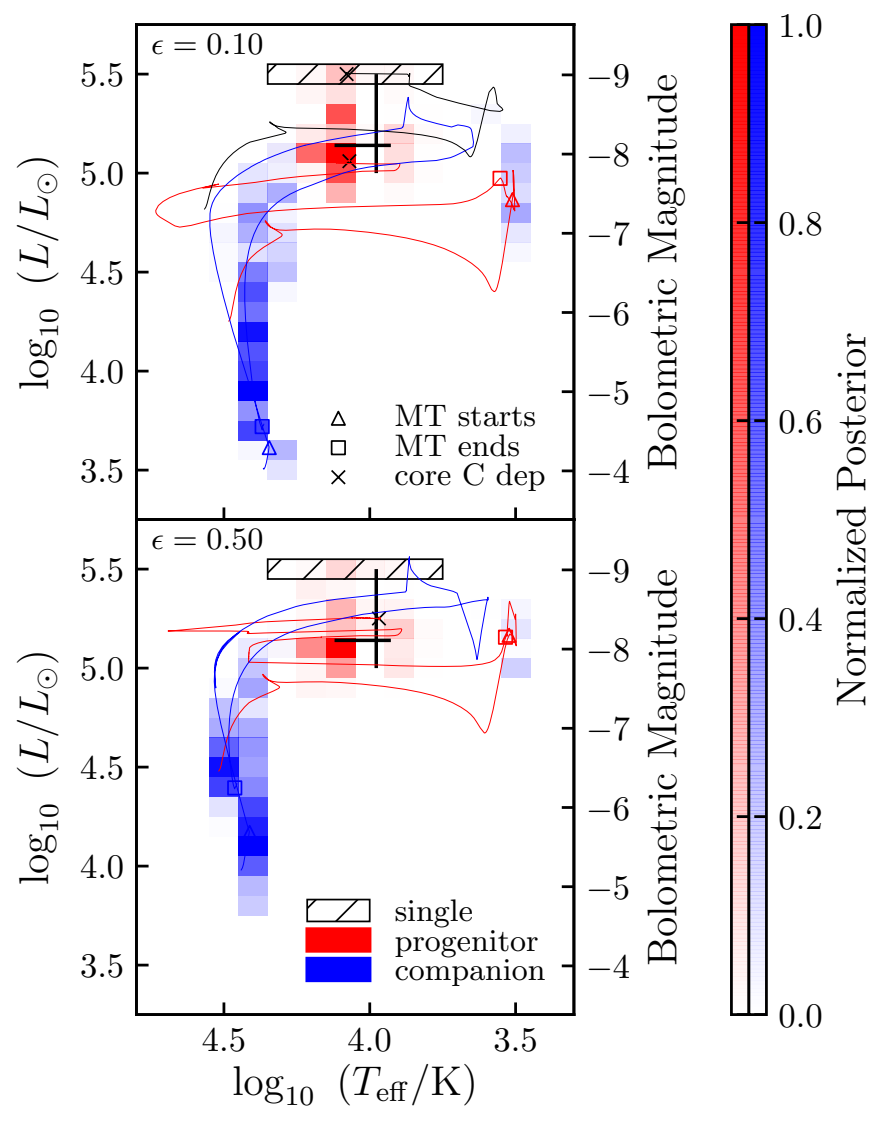

FIG. 3.- Distributions of H-R locations of potential single and binary $(\epsilon=0.1$ (top) and 0.5 (bottom)) star progenitors of SN 2016gkg. The red (blue) color scales show distributions for the primary (secondary) of binary star progenitors. Red (blue) H-R tracks are for the primary (secondary) of the binary star model with the highest posterior probability for the corresponding $\epsilon: M_{\mathrm{ZAMS}, 1}=$ $14.45(17.38) M_{\odot}, q_{\mathrm{ZAMS}} \equiv M_{\mathrm{ZAMS}, 2} / M_{\mathrm{ZAMS}, 1}=0.575(0.675)$, and $P_{\text {orb }}=2291$ (3311) days for $\epsilon=0.1$ (0.5). Mass transfer (MT) is defined to be taking place when mass transfer rate due to $\mathrm{RLO}$ is $\geq 10^{-6} M_{\odot} \mathrm{yr}^{-1}$. The black $\mathrm{H}-\mathrm{R}$ track is for the singlestar model with the highest posterior probability: $M_{\mathrm{ZAMS}}=23.28$ $M_{\odot}$. The hatched region shows H-R locations of potential singlestar progenitors with normalized posterior probabilities $\geq 0.01$. Black error-hairs show one-third of the $3 \sigma$ constraints on the observed progenitor for SN 2016gkg (Table 1). If present, the binary companion of SN $2016 \mathrm{gkg}$ has $M_{\mathrm{bol}} \gtrsim-8.5$ and is a main-sequence or red-giant star (for binary star progenitors with initial mass ratios $\sim 1)$. As such no strong constraints can be placed on the companion.

whose evolution largely resembles their single-star counterparts. There are fewer binary star progenitors with $\epsilon=0.5, q_{\text {ZAMS }}>0.7$, and $\log _{10}\left(P_{\text {orb }} / \mathrm{d}\right) \lesssim 3.1$ as they experience unstable mass transfer or evolve into contact, which lead to a merger.

In figure 2 we show the distribution of pre-SN properties of potential single and binary star progenitors of SN 2016gkg. The three peaks in the distributions of initial primary mass (Figure 1) of binary star progenitors roughly translate to the distributions of preSN hydrogen-envelope and helium-core mass. Pre-SN hydrogen-envelope and helium-core mass for potential binary star progenitors are clearly smaller than for potential single-star progenitors (e.g., Podsiadlowski et al. 1992, Yoon et al. 2010; Claeys et al. 2011) typically by
$0.1 M_{\odot}$ and $2 M_{\odot}$, respectively. Therefore, these can be used to distinguish progenitor scenarios. While progenitor helium-core mass constraints are currently unavailable for SN $2016 \mathrm{gkg}$, their existence could increase the likelihood of a binary progenitor of SN 2016gkg significantly by ruling out several single-star progenitors (see below for a discussion on rates). The distribution of all binary star properties shown in Figures 1 and 2 remain roughly the same regardless of whether or not we apply model-dependent progenitor hydrogen-envelope mass and radius constraints.

In figure 3 we show the distribution of locations on the Hertzsprung-Russell (H-R) diagram of potential single and binary star progenitors of SN 2016gkg. The luminosities of binary progenitors are smaller than of singlestar progenitors. This is a consequence of smaller pre-SN helium-core masses for binary progenitors (see Figure 2). The secondaries of binary progenitors mostly lie on the main-sequence and are less luminous than their primaries with $M_{\text {bol }} \gtrsim-8.5$. Some binary progenitors with initial mass ratios $\sim 1$ have evolved secondaries that are on the red-giant branch (RGB). We find that it is unlikely for the secondary to lie between the main sequence and the RGB, which is the case for the companion of SN 1993J (Maund et al. 2004). Otherwise, no strong constraints can be placed on binary companions' location on the H-R diagram. We also note that in binaries with secondaries of luminosities similar to that of the primary, flux from the secondary may contaminate flux from the primary, making our calculations inconsistent with the derivation of observed constraints. We find that the total posterior probability of progenitors having secondaries with luminosities within a factor of 2 of the primary (and thus potentially contaminated) is $\sim 12 \%$ for $\epsilon=0.1$ and $\sim 4 \%$ for $\epsilon=0.5$.

$\mathrm{X}$-ray/radio observations can be used to infer the CSM density around SN progenitors and thus trace the mass loss history of the progenitor star. We use our models to infer the CSM density at $10^{16} \mathrm{~cm}$ to compare with the results of Margutti et al. (2017) (example Figure $6)$. Our models have a SN Ibc-like mass loss history: $v_{\text {wind }} \sim 50-250 \mathrm{~km} \mathrm{~s}^{-1}$ and $\dot{M}_{\text {wind }} \sim 10^{-4.8}-10^{-6} M_{\odot}$ $\mathrm{yr}^{-1}$. This is because all potential binary progenitors detach before core-collapse. If future measurements indicate that SN 2016gkg also experienced high mass-loss rates $\left(\sim 10^{-4} M_{\odot} \mathrm{yr}^{-1}\right)$, like those for other Type IIb $\mathrm{SNe}$ in the aforementioned study, then it would indicate that the progenitor experienced a period of enhanced mass loss just before explosion.

Finally, we compute the probability that the progenitor of SN 2016gkg was a binary: the total posterior probability of all model binary star progenitors divided by total posterior probability of all model single and binary star progenitors. In Table 2 we list probabilities of a binary star progenitor of SN 2016gkg not applying and applying model-dependent progenitor hydrogen-envelope mass and radius constraints, for $f_{\text {bin }}=0.50$ and various values of $\alpha, \beta$, and $\gamma$. We find that the probability of a binary star progenitor of SN 2016gkg with $\epsilon=0.1$ and 0.5 not-given (given) progenitor hydrogen-envelope mass and radius constraints is $22 \%(44 \%)$ and $13 \%(28 \%$, respectively, for our fiducial values of $\alpha(2.3), \beta(-1.0)$, and $\gamma(-0.22)$. 
TABLE 2

Probability of A BINARY STAR PROGENITOR OF SN 2016GKG

\begin{tabular}{|c|c|c|c|c|c|c|}
\hline \multirow[t]{2}{*}{$\alpha$} & \multirow[t]{2}{*}{$\beta$} & \multirow[t]{2}{*}{$\gamma$} & \multicolumn{2}{|c|}{$P_{\text {binary }} \mid L, T_{\text {eff }}$} & \multicolumn{2}{|c|}{$P_{\text {binary }} \mid L, T_{\text {eff }}, M_{\text {env }}, R$} \\
\hline & & & $\epsilon=0.1$ & $\epsilon=0.5$ & $\epsilon=0.1$ & $\epsilon=0.5$ \\
\hline \multirow{6}{*}{1.6} & \multirow{2}{*}{-2.0} & 0.00 & 0.13 & 0.08 & 0.28 & 0.17 \\
\hline & & -0.22 & 0.11 & 0.06 & 0.25 & 0.15 \\
\hline & \multirow{2}{*}{-1.0} & 0.00 & 0.21 & 0.12 & 0.42 & 0.26 \\
\hline & & -0.22 & 0.18 & 0.11 & 0.38 & 0.23 \\
\hline & \multirow{2}{*}{0.0} & 0.00 & 0.29 & 0.17 & 0.52 & 0.34 \\
\hline & & -0.22 & 0.25 & 0.15 & 0.48 & 0.30 \\
\hline \multirow{6}{*}{2.3} & \multirow{2}{*}{-2.0} & 0.00 & 0.16 & 0.10 & 0.34 & 0.22 \\
\hline & & -0.22 & 0.14 & 0.08 & 0.31 & 0.19 \\
\hline & \multirow{2}{*}{-1.0} & 0.00 & 0.25 & 0.15 & 0.48 & 0.32 \\
\hline & & -0.22 & 0.22 & 0.13 & 0.44 & 0.28 \\
\hline & \multirow{2}{*}{0.0} & 0.00 & 0.34 & 0.21 & 0.59 & 0.40 \\
\hline & & -0.22 & 0.31 & 0.18 & 0.55 & 0.36 \\
\hline \multirow{6}{*}{3.0} & \multirow{2}{*}{-2.0} & 0.00 & 0.20 & 0.12 & 0.41 & 0.27 \\
\hline & & -0.22 & 0.17 & 0.11 & 0.37 & 0.24 \\
\hline & \multirow{2}{*}{-1.0} & 0.00 & 0.31 & 0.19 & 0.56 & 0.38 \\
\hline & & -0.22 & 0.28 & 0.17 & 0.52 & 0.35 \\
\hline & \multirow{2}{*}{0.0} & 0.00 & 0.41 & 0.25 & 0.66 & 0.47 \\
\hline & & -0.22 & 0.37 & 0.22 & 0.62 & 0.43 \\
\hline
\end{tabular}

Note. $-f_{\mathrm{bin}}=0.5$, and $\alpha, \beta$, and $\gamma$ are parameters for the priors on the initial mass, $\log _{10} M_{\mathrm{ZAMS}}$, initial mass ratio, $q_{\mathrm{ZAMS}}$, and initial orbital period, $P_{\mathrm{orb}}$, respectively (see Eqs. 6 7 , 7 and 8 .

\section{CONCLUSIONS}

We use Bayesian inference and a large grid of single and binary star models to derive the distributions of potential progenitors and companions of SN 2016gkg. We find that potential binary star progenitors have lower initial primary mass and pre-SN hydrogen-envelope and heliumcore mass than single-star progenitors. The probability that the progenitor of SN 2016gkg was a binary with $\epsilon=$ $0.1(0.5)$ is $22 \%(13 \%)$ if we only use luminosity and effective temperature constraints on the progenitor star. Applying model-dependent observational constraints on the progenitor hydrogen-envelope mass and radius rule out several single-star progenitors, favoring a binary as the progenitor of SN 2016gkg (44\% for $\epsilon=0.1$ and $28 \%$ for $\epsilon$ $=0.5)$. In either case, there is no clear preference for a binary star progenitor of SN $2016 \mathrm{gkg}$. This is in contrast to binaries being the currently favored progenitors of Type IIb SNe. We find that, a binary companion, if present, has $M_{\mathrm{bol}} \gtrsim-8.5$ and is a main-sequence or red-giant star. As such, we are unable to find strong constraints on the nature of the companion star. Constraints on the progenitor helium-core mass can help tighten constraints on the progenitor. Similarly, improved constraints on the progenitor luminosity can significantly narrow the parameter space for progenitors. We would like to stress that the parameter space for Type IIb SN progenitors is strongly dependent on the progenitor metallicity. At lower metallicities, the parameter space for binary progenitors of Type IIb SNe widens significantly (Yoon et al. 2017). We expect that the results presented here will differ strongly at low metallicities with the probability of a binary progenitor increasing significantly. Nevertheless, the probability of a binary progenitor derived at solar metallicity represents a lower limit. At lower metallicities, the binary star channel towards Type IIb $\mathrm{SNe}$ dominates for a couple of reasons. First, the parameter space for binary star Type IIb SN progenitors widens significantly at lower metallicity (Yoon et al. 2017). Second, single star Type IIb SN progenitors are produced at higher masses (due to the scaling in wind mass-loss) and are thus disfavored by the IMF. A detailed investigation into the effects of metallicity on the relative importance of single and binary progenitors of Type IIb SNe would be an interesting line of future investigation.

NS, PM, and VK acknowledge support from NSF grant AST-1517753. NS acknowledges support from NSF grant DGE-1450006 and from Northwestern University. This work was performed in part at the Aspen Center for Physics, which is supported by National Science Foundation through grant PHY-1607611. This research was supported by computational resources provided for the Quest high performance computing facility at Northwestern University which is jointly supported by the National Science Foundation through grant NSF PHY-1126812, the Office of the Provost, the Office for Research, and Northwestern University Information Technology.

\section{REFERENCES}

Aldering, G., Humphreys, R. M., \& Richmond, M., 1994, AJ, 107, 662

Arcavi, I., Hosseinzadeh, G., Brown, P. J., et al. 2017, ApJ, 827, L2

Arnett, W. D., \& Meakin, C. 2011, ApJ, 741, 33

Brott, I., de Mink, S. E., Cantiello, M., et al. 2011, A\&A, 530, A115

Claeys, J. S. W., de Mink, S. E., Pols, O. R., et al. 2011 A\&A, 528, A131

de Jager, C., Nieuwenhuijzen, H., \& van der Hucht, K. A. 1988, A\&AS, 72, 259

Folatelli, G., Bersten, M. C., Benvenuto, O. G., et al. 2014, ApJ, 793, L22

Folatelli, G., Bersten, M. C., Kuncarayakti, H., et al. 2015, ApJ, 811,147

Fox, O. D., Azalee Bostroem, K., Van Dyk, S. D., et al. 2014, ApJ, 790, 17

Fuller, J. 2017, MNRAS, 470, 1642
Groh, J. H., Meynet, G., Georgy, C., et al. 2013, A\&A, 558, A131

Iglesias, C. A., \& Rogers, F. J. 1996, ApJ, 464, 943

Kilpatrick, C. D., Foley, R. J., Abramson, L. E., et al. 2017, MNRAS, 465, 4650

Kobulnicky, H. A., Kiminki, D. C., Lundquist, M. J., et al. 2014, ApJS, 213, 34

Kolb, U., \& Ritter, H. 1990, A\&A, 236, 385

Margutti, R., Chornock, R., Metzger, B. D., et al. 2017, ArXiv e-prints, 1704.05865

Maund, J. R., Smartt, S. J., Kudritzki, R. P., et al. 2004, Nature, 427,129

Maund, J. R., Fraser, M., Ergon, M., et al. 2011, ApJ, 739, L37

Moriya, T. J., Pruzhinskaya, M. V., Ergon, M., et al. 2016, MNRAS, 455, 423

Nugis, T., \& Lamers, H. J. G. L. M. 2000, A\&A, 360, 227

Paxton, B., Bildsten, L., Dotter, A., et al. 2011, ApJS, 192, 3

Paxton, B., Cantiello, M., Arras, P., et al. 2013, ApJS, 208, 4

Paxton, B., Marchant, P., Schwab, J., et al. 2015, ApJS, 220, 15 
Podsiadlowski, P., Joss, P. C., \& Hsu, J. J. L. 1992, ApJ, 391, 246

Podsiadlowski, P., Hsu, J. J. L., Joss, P. C., et al. 1993, Nature, 364, 509

Quataert, E., \& Shiode, J. 2012, MNRAS, 423, L92

Salpeter, E. E. 1955, ApJ, 121, 161

Shiode, J. H., Quataert, E., Cantiello, M., et al. 2013, MNRAS, 430,1736

Shiode, J., \& Quataert, E. 2014, ApJ, 780, 96

Smartt, S. J. 2009, ARA\&A, 47, 63

Smith, N., \& Arnett, W. D. 2014, ApJ, 785, 82

Smith, N. 2014, ARA\&A, 52, 487

Soker, N. 2017, MNRAS, 470, L102

Stancliffe, R. J., \& Eldridge, J. J. 2009, MNRAS, 396, 1699
Tartaglia, L., Fraser, M., Sand, D. J., et al. 2017, ApJ, 836, L12

Van Dyk, S. D., Li, W., Cenko, S. B., et al. 2011, ApJ, 741, L28

Van Dyk, S. D., Zheng, W., Fox, O. D., et al. 2014, AJ, 147, 37

Vink, J. S., de Koter, A., \& Lamers, H. J. G. L. M. 2001, A\&A, 369,574

Woosley, S. E., Eastman, R. G., Weaver, T. A., et al. 1994, ApJ, 429,300

Woosley, S. E., Heger, A., \& Weaver, T. A. 2002, Reviews of Modern Physics, 74, 1015

Yoon, S.-C., Langer, N., \& Norman, C. 2006, A\&A, 460, 199

Yoon, S.-C., Woosley, S. E., \& Langer, N. 2010, ApJ, 72, 940

Yoon, S.-C., Dessart, L., \& Clocchiatti, A. 2017, ApJ, 840, 10 